\title{
A Novel Channel Estimation Technique for Complexity Reduction of Least Minimum Mean Square Error
}

\author{
${ }^{1}$ Hany Mohamed El-Ansary, \\ ${ }^{1}$ Hussein Ghouz, ${ }^{1}$ Ashraf Mamdouh Aziz and ${ }^{2}$ Gamal Mabrouk \\ ${ }^{1}$ Department of Communications Engineering, Faculty of Engineering, \\ Arab Academy for Science, Technology Maritime Transport University, Cairo, Egypt \\ ${ }^{2}$ Department of Communications Engineering, Faculty of Engineering, Military Technical University, Cairo, Egypt
}

Received 2013-07-26, Revised 2013-08-23; Accepted 2013-08-28

\begin{abstract}
Channel estimation technique is to predict the frequency response of the radio channel using pilot signal. Channel estimation technique is important part of LTE receiver design. Such technique is used to recover the transmitted signal correctly and to know how the channel conditions are changed over frequency and time. Each channel estimator has a specified complexity computed according to its mathematical model. Complexity Reduction of any channel estimator is very important to achieve high performance. This paper studied in details the performance enhancement of LMMSE channel estimation technique in the downlink of LTE system. The proposed algorithm is based on Roza Zheng channel model and the matrix transpose rather than matrix inverse. This dramatically reduces the complexity of LMMSE. In adition, the complexity of the proposed algorithm is invariant with the number of multipath components in case of fast fading channels. Moreover, the received signal quality has been improved as compared to the LS. The processing time of the proposed algorithm is reduced by using new criteria of auto correlation. The performance of the proposed algorithm has been evaluated using the mean squared error as the performance metric. Simulation results showed that the MSE is reduced by $89.5 \%$ for LS and by $38.7 \%$ for LMMSE. Finally, the bit error rate has been enhanced using the proposed algorithm.
\end{abstract}

Keywords: LTE System, Channel Estimation, OFDM System, MIMO System

\section{INTRODUCTION}

Wireless multimedia services require a high speed data access in order to satisfy their exponential growing. Therefore, various techniques have been proposed in recent years to achieve high system capacities. The Multiple-Input Multiple Output MIMO concept has attracted a lot of attention in wireless communications due to its potential to increase the system capacity without extra bandwidth (Rana, 2010). Multipath propagation usually causes selective frequency channels, to combat the effect of frequency selective fading,
MIMO is associated with Orthogonal FrequencyDivision Multiplexing (OFDM) technique. OFDM is a modulation technique which transforms frequency selective channel into a set of parallel flat fading channels. A cyclic prefix $\mathrm{CP}$ is added at the beginning of each OFDM symbol to eliminate ICI and ISI. However, the inserted cyclic prefix $\mathrm{CP}$ length is assumed to be equal or longer than the maximum propagation delay of the channel. But in some cases because of some unforeseen channel behaviour, the cyclic prefix can be shorter than propagation delay of the channel. In this case, both ICI and ISI occured this makes the task of

Corresponding Author: Hany Mohamed El-Ansary, Department of Communications Engineering, Faculty of Engineering, Arab Academy for Science, Technology Maritime Transport University, Cairo, Egypt 
channel estimation more difficult (Jing and Fang, 2011). Equalization techniques that could flexibly detect the signals in both cases in MIMO-OFDM systems are discussed in (Schwarz et al., 2010; Ketonen et al., 2009). The 3GPP Long Term Evolution (LTE) defining the next generation radio access network. LTE Downlink systems adopt Orthogonal Frequency Division Multiple Access (OFDMA) MIMO to provide up to $100 \mathrm{Mbps}$ (assuming a $2 \times 2$ MIMO system with $20 \mathrm{MHz}$ bwidth). The performance of a MIMO-OFDM communication system significantly depends upon the channel estimation. Channel estimation techniques for MIMO-OFDM systems like LS and LMMSE (Jiang et al., 2010; Deng and Wang, 2011).

The performance evaluation of the two channel estimation techniques: Least Square (LS) Least Minimum Mean Square Error (LMMSE) are discussed in (Hou and Liu, 2010; Chen et al., 2011) but many works still required for studying the performance evaluation of those two estimators in LTE downlink under the effect of channel propagation delay to enhance the signal quality of LS estimator in the same time reduce the complexity of the LMMSE estimator (Song et al., 2011).

This paper introduce a proposed algorithm for channel estimation in downlink LTE. The proposed algorithm has less complexity than the LS algorithm due to using matrix transpose rather than matrix inverse. The proposed algorithm has less BER than LMMSE as the matrix A doesn't contain all data of the user but it contain neighbour's users only. The time correlated channel was generated by an implementation of the Rosa Zheng model. Also the LTE is asynchronized system so the proposed algorithm improves this synchronization prossecc. raising the number of subcarriers $\mathrm{N}_{\mathrm{fft}}$ make reduction in MSE, reduction the error that produced from non-orthogonality, reduction the AWGN and reduction all interferences signals. The proposed algorithm has some sources of Errors like possible imperfect orthogonality of the used carries, Additive White Gaussian Noise (AWGN) Quantization of the channel delay.

This paper is organized as following: section 2 present the system and channel model, section 3 present the proposed channel estimation algorithm, section 4 present the simulation results, section 5 present the conclusion.

\section{SYSTEM AND CHANNEL MODEL}

\subsection{System Model}

OFDM system block diagram consits of encoding, scrambling...,. A discrete-time domain OFDM signal for one symbol interval can be expressed as Equation 1:

$$
x_{n}=\frac{1}{N_{f f t}} \sum_{k=1}^{N_{f f t}-1} X_{k} e^{j \frac{2 \pi k n}{N_{f f t}}}
$$

where, $\mathrm{x}_{\mathrm{n}}$ is the transmitted symbol, $\mathrm{n}$ denotes the discretetime index $0 \leq \mathrm{n} \leq \mathrm{N}_{\mathrm{fft}}-1, \mathrm{~N}_{\mathrm{fft}}$ is the duration of one OFDM symbol interval in samples, the number of subcarriers is also $\mathrm{N}_{\mathrm{fft}}, \mathrm{K}$ represents the discrete-frequency domain index of the $\mathrm{K}^{\text {th }}$ sub-carrier. In most OFDM systems, it is commonly to insert Cyclic Prefix (CP) of $\mathrm{N}_{\mathrm{g}}$ samples length before the data symbol as a guard interval to prevent Inter-Symbol Interference (ISI) Equation 2:

$\mathrm{X}_{\mathrm{n}}=\frac{1}{\mathrm{~N}_{\mathrm{fft}}} \sum_{\mathrm{k}=1}^{\mathrm{N}_{\mathrm{fft}}-1} \mathrm{X}_{\mathrm{k}} \mathrm{e}^{\mathrm{j} \frac{2 \pi \mathrm{kn}}{\mathrm{N}_{\mathrm{fft}}}}, \ldots \ldots . .-\mathrm{N}_{\mathrm{g}}<\mathrm{n}<\mathrm{N}_{\mathrm{fft}}-1$

Or in matrix form as Equation 3:

$$
\mathrm{x}_{\mathrm{n}}=\frac{1}{\mathrm{~N}_{\mathrm{fft}}} \mathrm{Fx}_{\mathrm{k}}
$$

where, $x_{k} \in C^{N_{\text {fit }} \times 1}$ are the transmitted vectors, $F$ is the inverse fast fourier fransform (IFFT) matrix operator $\mathrm{F} \in \mathrm{C}^{\mathrm{N}_{\mathrm{fft}} \times \mathrm{N}_{\mathrm{fft}}}$ and defined as Equation 4:

$\mathrm{F}=\sum_{\mathrm{k}=1}^{\mathrm{N}_{\mathrm{fft}}-1} \mathrm{e}^{\mathrm{j} \frac{2 \pi \mathrm{kn}}{N_{\mathrm{ftt}}}}, 0 \leq \mathrm{k}, \mathrm{n} \leq \mathrm{N}_{\mathrm{fft}}-1$

\subsection{Channel Model}

For the convenience of analysis, the channel impulse response of any multipath channel can be re-written by introducing Multi Path Components (MPCs) that represented as follows Equation 5:

$$
h(t)=\sum_{1=0}^{N_{p}-1} \alpha_{1} \delta\left(t-\tau_{1}\right)
$$

where, $h(t)$ is the channel response, $N_{p}$ is the total number of MPCs, $\alpha_{\mathrm{L}} \tau_{\mathrm{L}}$ area the path attenuationthe corresponding delay of any received MPC, respectively, $l$ is the index of this path. The channel weights $\alpha_{L}$ are assumed to be complex in general. This allows the CIR of the RF channel to be expressed in a general form that is compatible with any multipath channel profile regardless of the nature of the amplitude delay statistics. Also, for convenience of analysis, the whole time duration of the CIR is divided into time bins 
i.e., let $\tau_{1}=1 \Delta \tau$ and $\mathrm{I}=0,1, \ldots, \mathrm{N}_{\mathrm{p}}-1,1, \ldots, \mathrm{N}_{\mathrm{p}}-1$ where $\Delta \tau$ is the timing resolution. This time resolution is assigned to the symbol duration in the process of channel estimation. According to the timing resolution limitation of either the receiver sampling or the bandwidth of the pulse, $\Delta \tau$ is selected in simulations, so no more than one MPC arrives in this minimum timing resolution. This last form of the CIR suggests that the channel can be represented as an FIR filter as illustrated in Figure 1.

It can be intuitively thought that the FIR model of the physical RF channel is a well sampled theoretical version of the physical channel model for the same channel. In this paper, it is assumed that the maximum delay of multipath channel is smaller than guard interval, which means no ISI occurs. The ROSA model is new sum-of-sinusoids statistical simulation models which are proposed for Rayleigh fading channels. These new models can rom path gain, rom initial phase, conditional rom Doppler frequency for all individual sinusoids. It is shown that the autocorrelations and cross correlations of the quadrature components, the autocorrelation of the complex envelope of the new simulators match the desired ones exactly, even if the number of sinusoids is as small as a single-digit integer. Moreover, the probability density functions of the envelope phase, the level crossing rate, the average fade duration, the autocorrelation of the squared fading envelope which contains fourth order statistics of the new simulators, asymptotically approach the correct ones as the number of sinusoids approaches infinity, while good convergence is achieved even when the number of sinusoids is as small aseight. The new simulators can be directly used to generate multiple uncorrelated fading waveforms for frequency selective fading channels, multiple-input multiple-output channels, diversity combining scenarios. Statistical properties of one of the new simulators are evaluated by numerical results, finding good agreements.

Consider a frequency-nonselective fading channel comprised of $\mathrm{N}_{\mathrm{p}}$ propagation paths, the low-pass fading process is given by Equation 6:

$$
\left.g(t)=E_{0} \sum_{l=1}^{N_{p}} C_{n} e^{\left[j\left(w_{d} t \cos \alpha_{n}+\phi_{n}\right)\right.}\right]
$$

where, $\mathrm{E}_{0}$ is a scaling constant $\mathrm{C}_{\mathrm{n}}, \alpha_{\mathrm{n}}$ and $\phi_{\mathrm{n}}$ are, respectively, the rom path gain, angle of incoming wave, initial phase associated with the $1^{\text {th }}$ propagation path, $\mathrm{w}_{\mathrm{d}}$ is the maximum radian Doppler frequency occurring when $\alpha_{n}=0$.

\subsection{Receiver Signal Model}

At the receiver side, the received signal over multipath time-varying channel after removing the guard interval can be expressed as Equation 7:

$\mathrm{y}_{\mathrm{n}}(\mathrm{t})=\sum_{\mathrm{l}=0}^{\mathrm{N}_{\mathrm{p}}-1}\left(\mathrm{~h}_{\mathrm{n}}^{(1)} \mathrm{x}_{\mathrm{n}-\tau(\mathrm{l})}\right)(\mathrm{t})+\eta(\mathrm{t})$

where, $y_{n}(t)$ is the received symbol, $\eta(t)$ Gaussian noise. A Low Pass Filter (LPF) is needed in the demodulation process, implemented as an integrator whose integration interval spans an OFDM symbol duration:

$$
\int_{t_{o}}^{t_{o}+T_{s}} y_{n}(t) d t=\int_{t_{o}}^{t_{o}+T_{s}}\left(\sum_{l=0}^{N_{p}-1}\left(h_{n}^{(l)} x_{n-\tau(l)}\right)(t)+\eta(t)\right) d t
$$

The integral in Equation (8) is then sampled prior to applying a Fast Fourier Transform (FFT) operation. An observation vector $\mathrm{y}_{\mathrm{n}}$ is formed on a symbol by symbol using collecting together $\mathrm{N}_{\mathrm{fft}}$ successive output samples corresponding to a time interval of OFDM symbol duration (Aziz, 2011). This observation vector can be written in matrix form as Equation 9:

$\mathrm{y}_{\mathrm{n}}=\mathrm{A}_{\mathrm{n}}+\mathrm{w}_{\mathrm{n}}$

where, $A_{n}$ is the channel parameters matrix $A_{n} \in C^{N_{\text {Nin }} \times 1}$ $\mathrm{w}_{\mathrm{n}}$ is a realization vector of the sampled AWGN rom process $n(t)$. Extracting the channel parameters from the matrix $A_{n}$ is a difficult task as the matrix $A_{n}$ is in general complex for the processing system as it includes the user data symbols, the pilot sequence that help in channel estimation process, the unknown channel parameters (Aziz et al., 2011). To facilitate the process of estimation of the unknown channel parameters it is necessary to decompose $A_{n}$ into the product of two independent matrices as Equation 10:

$\mathrm{y}_{\mathrm{n}}=\mathrm{H}_{\mathrm{n}} \mathrm{x}_{\mathrm{n}}+\mathrm{w}_{\mathrm{n}}$

where, $H_{n}$ is the channel matrix, $x_{n} \in C^{N_{\mathrm{ft}} \times 1}$ is the information bearing vector. In Equation (8), assuming that the system is generally asynchronous, that is, the OFDM receiver has an arbitrary timing reference which is not necessarily to aligned with the OFDM symbol boundaries, thus the integration period over one symbol may in general overlap two adjacent received symbols, the actual receiving situation of non integer delayed symbol sequence. 


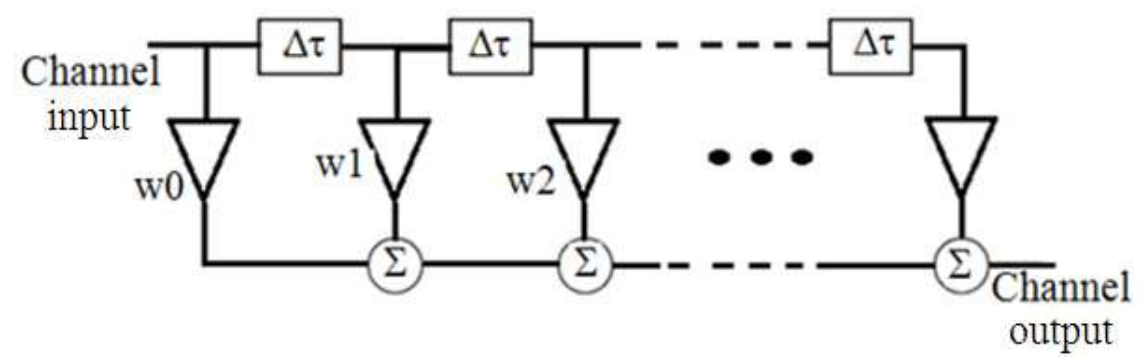

Fig. 1. The FIR filter model of a multipath channel

Each sample can be viewed as composed of a linear combination of the values of these two symbols, that is, the past and the current symbols $X_{n}, X_{n-1}$ Equation 11 and 12 :

$$
\begin{aligned}
& \int_{\mathrm{t}_{\mathrm{o}}+\mathrm{n} \mathrm{T}_{\mathrm{S}}}^{\mathrm{t}_{\mathrm{o}}+(\mathrm{n}+1) \mathrm{T}_{\mathrm{s}}} \mathrm{y}_{\mathrm{n}}(\mathrm{t}) \mathrm{dt}=\int_{\mathrm{t}_{\mathrm{o}}+\mathrm{nT} \mathrm{T}_{\mathrm{s}}}^{\mathrm{t}_{\mathrm{o}}+(\mathrm{n}+1) \mathrm{T}_{\mathrm{s}}}\left(\sum_{\mathrm{l}=0}^{\mathrm{N}_{\mathrm{p}}-1}\left(\mathrm{~h}_{\mathrm{n}}^{(1)} \mathrm{x}_{\mathrm{n}-\tau(1)}\right)(\mathrm{t})+\eta(\mathrm{t})\right) \mathrm{dt} \\
& \int_{\mathrm{t}_{\mathrm{o}}+\mathrm{n} \mathrm{T}_{\mathrm{S}}}^{\mathrm{t}_{\mathrm{o}}+(\mathrm{n}+1) \mathrm{T}_{\mathrm{S}}} \mathrm{y}_{\mathrm{n}}(\mathrm{t}) \mathrm{dt}=\mathrm{I}_{\mathrm{n}}^{L}+\mathrm{I}_{\mathrm{n}}^{R}+\mathrm{w}_{\mathrm{n}}
\end{aligned}
$$

where, $\mathrm{I}_{\mathrm{n}}^{\mathrm{L}}$ is the part of the integral that yields the value of the integration over $\varsigma T_{s}$ and $I_{n}^{R}$ is the part of the integral that yields the value of the integration over $(1-\varsigma) T_{s}$. Consequently the observation vector $y_{n}$ is a super position of two exclusive vectors $a_{n}^{R}, a_{n}^{L}$ Equation 13:

$$
\mathrm{y}_{\mathrm{n}}=\left[\mathrm{a}_{\mathrm{n}}^{\mathrm{R}}+\mathrm{a}_{\mathrm{n}}^{\mathrm{L}}\right]+\mathrm{W}_{\mathrm{n}}
$$

As the sampler is a discrete time device, the time delays of the channel is quantized by normalization to the sampling interval which is the same as the OFDM symbol duration $\mathrm{T}_{\mathrm{s}}$ hence Equation 14-16:

$$
\frac{\tau_{\mathrm{p}}}{\mathrm{T}_{\mathrm{s}}}=\mathrm{q}+\zeta, \mathrm{q} \in\left\{0,1, \ldots, \mathrm{N}_{\mathrm{FFT}}-1\right\}, \zeta \in[0,1)
$$

$I_{n}^{R}=\sum_{l=0}^{N_{p}-1}\left(\begin{array}{c}\alpha_{l}(1-\zeta) x_{n} \int_{t_{o}+n T_{s}}^{t_{o}+(n+1) T_{s}} y_{n}(t) c_{n}\left(t-q T_{s}\right) d t+\alpha_{l} \zeta x_{n-1} \\ \int_{t_{o}+n T_{s}}^{t_{o}+(n+1) T_{s}} y_{n}(t) c_{n}\left(t-q T_{s}\right) d t\end{array}\right)$

$$
I_{n}^{L}=\sum_{l=0}^{N_{p}-1}\left(\begin{array}{c}
\alpha_{l}(1-\zeta) x_{n} \int_{t_{t_{o}+n T_{s}}}^{t_{o}+(n+1) T_{s}} y_{n}(t) c_{n}\left(t+q T_{s}\right) d t+\alpha_{l} \zeta x_{n-1} \\
\int_{t_{o}+n T_{s}}^{t_{o}+(n+1) T_{s}} y_{n}(t) c_{n}\left(t+q T_{s}\right) d t
\end{array}\right)
$$

where, $\mathrm{q}$ is the integer number of the part of the symbol, $\zeta$ is the fraction number of the part of the symbol. The contribution of the $n^{\text {th }}$ subcarrier $C_{n}(t)$ in the vector $y_{n}$ appears in two parts, the right part of the OFDM symbol shown as $\mathrm{R}$ appears in the left symbol $\mathrm{x}_{\mathrm{n}-1}$ the left part shown as L appears in the right symbol $x_{n}$. The weights for the linear combination of the two vectors that contribute to the right part of symbols $x_{n}$ are $\zeta, 1-\zeta$. The matrices $U_{n}^{R} \in C^{N_{F F T} \times N_{F F T}}$ and $U_{n}^{L} \in C^{N_{F F T} \times N_{F F T}}$ are formed by arranging columns of $\mathrm{C}\left(\mathrm{t}-\mathrm{qT}_{\mathrm{s}}\right), \mathrm{C}\left(\mathrm{t}+\mathrm{qT}_{\mathrm{s}}\right)$ representing the orthogonal carriers defined on $0 \leq \mathrm{t} \leq \mathrm{T}_{\mathrm{s}}$ delayed by all possible integer delays q. $U_{n}^{R}, U_{n}^{L}$ can be written in Equation 17 and 18:

$$
\begin{aligned}
& \mathbf{U}_{n}^{L}[q]=\left[\begin{array}{ll}
\mathbf{c}_{n}^{L}[0] & \mathbf{c}_{n}^{L}[1] \ldots \mathbf{c}_{n}^{L}\left[N_{f f t}-1\right]
\end{array}\right] \\
& \mathbf{U}_{n}^{R}[q]=\left[\mathbf{c}_{n}^{R}[0] \mathbf{c}_{n}^{R}[1] \ldots \mathbf{c}_{n}^{R}\left[N_{f f t}-1\right]\right]
\end{aligned}
$$

where, $c_{n}^{L, R}[q]$ is the $n^{\text {th }}$ subcarrier $C_{n}(t)$ representing either the left or right cyclically shifted by q samples. Let $\mathrm{Z}_{\mathrm{n}} \mathrm{K} \in \mathrm{C}^{\mathrm{N}}{ }_{\mathrm{fft}}{ }^{\times 1}$ be the composite channel impulse response vector sampled $\mathrm{N}_{\mathrm{fft}}$ times over $\mathrm{T}_{\mathrm{s}}$ that consisting of all the weight vectors in the linear combination of the vectors $c_{n}^{L, R}[q]$, while the vector $Z_{n}$ is expressed as follows Equation 19-21:

$$
\mathrm{z}_{\mathrm{n}}=[0 \ldots \mathrm{M} \ldots 0]
$$


$\mathrm{M}=\left[\alpha_{1}(1-\zeta) \alpha_{1} \zeta \alpha_{\mathrm{N}_{\mathrm{p}}}(1-\zeta) \alpha_{\mathrm{N}_{\mathrm{p}}} \zeta\right]$

$\mathrm{y}_{\mathrm{n}}=\left[\mathrm{U}_{\mathrm{n}}^{\mathrm{R}} \quad \mathrm{U}_{\mathrm{n}}^{\mathrm{L}}\right]\left(\mathrm{z}_{\mathrm{n}} \odot \mathrm{x}_{\mathrm{n}}\right)+\mathrm{w}_{\mathrm{n}}=\left[\mathrm{U}_{\mathrm{n}}^{\mathrm{R}} \mathrm{z}_{\mathrm{n}} \mathrm{U}_{\mathrm{n}}^{\mathrm{L}} \mathrm{z}_{\mathrm{n}}\right] \mathrm{x}_{\mathrm{n}}+\mathrm{w}_{\mathrm{n}}$

where, $(\odot)$ is defined as the multiplication operator. The columns of matrix A can be expressed in terms of $\mathrm{U}_{\mathrm{n}}^{\mathrm{R}}, \mathrm{U}_{\mathrm{n}}^{\mathrm{L}}$ and $\mathrm{Z}_{\mathrm{n}}$ as Equation 22 and 23:

$a_{n}^{R}=U_{n}^{R} Z_{n}$

$\mathrm{a}_{\mathrm{n}}^{\mathrm{L}}=\mathrm{U}_{\mathrm{n}}^{\mathrm{L}} \mathrm{Z}_{\mathrm{N}}$

The received observation vector $\mathrm{y}_{\mathrm{n}}$ in Equation 9 can be writen by Equation 24:

$\mathrm{y}_{\mathrm{n}}=\left[\mathrm{U}_{\mathrm{n}}^{R} \mathrm{Z}_{\mathrm{n}} \mathrm{U}_{\mathrm{n}}^{L} \mathrm{Z}_{\mathrm{n}}\right] \mathrm{x}_{n}+\mathrm{w}_{n} ; \mathrm{x}_{n}=\left[\begin{array}{ll}x_{n} & x_{n-1}\end{array}\right]^{\mathrm{T}}$

where, $[.]^{\mathrm{T}}$ is the matrix transpose operator.

\section{THE PROPOSED CHANNEL ESTIMATION ALGORITHM}

The mathematical model for the received signal of the intended user should be used to extract the channel parameters like the attenuations and the corresponding delays. The proposed algorithm has the advantage of being consistent with a general multipath channel. The vector entries $\mathrm{x}_{n}, \mathrm{x}_{n-1}$ represent either the information or training symbols. Assuming $\mathrm{x}_{n}=1$ during the estimation proccess so that $\mathrm{y}_{\mathrm{n}}$ includes only the channel coefficients (Hong-Jin and Li-Fa, 2011). This assumption has the advantage that no specific form for the pilot sequence is required. The process of channel estimation is done by only periodic transmission of a linear combination of all carriers. These carriers have equal amplitudes for a number of times equal to the length of the pilot sequence in symbols. For only one symbol duration Equation (24) can now be expressed as follows Equation 25:

$\mathrm{y}_{\mathrm{n}}=\left[\mathrm{U}_{\mathrm{n}}^{\mathrm{R}}+\mathrm{U}_{\mathrm{n}}^{\mathrm{L}}\right] \mathrm{z}_{\mathrm{n}}+\mathrm{w}_{\mathrm{n}}$

Or equivalently Equation 26:

$\mathrm{y}_{\mathrm{n}}=\left[\mathrm{U}_{\mathrm{n}}^{\mathrm{R}}+\mathrm{U}_{\mathrm{n}}^{\mathrm{L}}\right] \mathrm{z}_{\mathrm{n}}+\mathrm{w}_{\mathrm{n}}$
Multiplying both sides of Equation 26 by $\mathrm{U}_{\mathrm{n}}^{T}$ :

$$
\mathrm{U}_{\mathrm{n}}^{\mathrm{T}} \mathrm{y}_{\mathrm{n}}=\mathrm{U}_{\mathrm{n}}^{\mathrm{T}} \mathrm{U}_{\mathrm{n}} \mathrm{z}_{\mathrm{n}}+\mathrm{U}_{\mathrm{n}}^{\mathrm{T}} \mathrm{w}_{\mathrm{n}}
$$

The product $\mathrm{U}_{\mathrm{n}}^{\mathrm{T}} \mathrm{U}_{\mathrm{n}}$ is a $\mathrm{N}_{\mathrm{fft}} \times \mathrm{N}_{\mathrm{fft}}$ matrix whose elements are the values of the autocorrelation function of the subcarrier matrix evaluated at different discrete time that has shifts ranging from 0 to $\mathrm{N}_{\mathrm{fft}}-1$ thus Equation 28:

$$
\mathrm{U}_{\mathrm{n}}^{\mathrm{T}} \mathrm{U}_{\mathrm{n}}=\mathrm{R}
$$

where, $\mathrm{R}$ is a Toeplitz autocorrelation matrix with the entry $(i, j)$ of the matrix $R$ is defined as follows Equation 29:

$$
\mathrm{R}_{\mathrm{ij}}=\mathrm{c}^{\mathrm{J}}[\mathrm{i}] \mathrm{c}[\mathrm{j}]
$$

where, $c^{T}[j]$ is a vector of the $k^{\text {th }}$ sampled sub-carrier shifted by i samples and $\mathrm{c}[\mathrm{J}]$ is at the same carrier shifted version of the same vector shifted by $J$ samples, thus the value of $R_{i j}$ is equal to $\mathrm{N}_{\mathrm{fft}}$ times $\delta_{\mathrm{ij}}$, the Kronicker delta function, if only the carriers are perfect orthogonal, however, for purpose of generalization, no need to assume that perfect orthogonality, assuming that the used carriers violates the orthogonality property due to a Doppler shift,the autocorrelation matrix $\mathrm{R}$ can be considered as consisting of the sum of two matrices, the identity matrix I scaled by $\mathrm{N}_{\mathrm{fft}}$ results if the carriers are a perfect orthogonal. Error matrix E appears only with the non orthogonal part as follows Equation:

$\mathrm{R}=\mathrm{U}_{\mathrm{n}}^{T} \mathrm{U}_{\mathrm{n}}=\mathrm{N}_{\mathrm{fft}}(\mathrm{I}+\mathrm{E})$

where, the entry $(i, j)$ of the matrix $E$ is expressed as follows Equation 31:

$E_{i j}=\left\{\begin{array}{cc}\frac{c^{T}[i] c[j]}{N_{f f t}}, & i=j \\ 0 & i=j\end{array}\right.$

The diagonal elements of the matrix $\mathrm{E}$ are always equal to zeroas. The number of these orthogonal subcarriers $\mathrm{N}_{\mathrm{fft}}$ are large enough to assume that the product $\mathrm{U}_{\mathrm{n}}^{\mathrm{T}} \mathrm{U}_{\mathrm{n}}$ can be well approximated by an identity matrix multiplied by $\mathrm{N}_{\mathrm{fft}}$.

$$
\operatorname{Lim}_{N_{\text {fft }} \rightarrow \infty} E=O
$$


where, $\mathrm{O}$ being the zero matrix, so Equation 27 can be written as:

$$
\lim _{\mathrm{N}_{\mathrm{ft}} \rightarrow \infty} \frac{\mathrm{U}_{\mathrm{n}}^{\mathrm{T}} \mathrm{y}_{\mathrm{n}}}{\mathrm{N}_{\mathrm{fft}}}=\mathrm{z}_{\mathrm{n}}
$$

The limit in Equation 33 can be replaced practically by using a very large number of the orthogonal subcarriers however this necessitates raising the limit from Equation 33:

$$
\frac{1}{N_{f f t}}\left(U_{n}^{T} y_{n}\right)=z_{n}+z_{n} E_{n}+\frac{1}{N_{f f t}}\left(U_{n}^{T} W_{n}\right)
$$

The LHS of Equation 34 is the estimate of the CIR vector $Z_{n}$ appearing on the RHS. Let's define the estimated CIR vector $\hat{z}_{n}$ as follows Equation 35:

$$
\hat{\mathrm{z}}_{\mathrm{n}}=\frac{1}{\mathrm{~N}_{\mathrm{fft}}}\left(\mathrm{U}_{\mathrm{n}}^{\mathrm{T}} \mathrm{U}_{\mathrm{n}} \mathrm{x}_{\mathrm{n}}\right)+\mathrm{e}_{\mathrm{n}}
$$

where, $e_{n}$ is the error vector defined in Equation (36):

$$
e_{n}=E_{n}+\frac{1}{N_{f f t}}\left(U_{n}^{T} W_{n}\right)
$$

This error is induced by one or more of three sources like imperfect orthogonality of the received sub-carriers, Doppler shift resulting from mobility and the additive white Gaussian noisethe quantization of the channel delays.

\section{SIMULATION RESULTS}

The simulation results are discussed the performance of the different channel estimation techniques while the MSE performance of the proposed channel estimation algorithm is compared with the optimal LMMSE estimation technique and the low rank LS estimation techniques (Zhao et al., 2010). The three techniques are performed in the frequency domain as it is well known that time domain channel estimation leads to the problem of propagating errors due to the back and forth FFT transformation that may lead to residual errors. Simulation parameters that used to evaluate the system performance are shown below in Table 1. The mean squared error is the performance metric of interest that is used to evaluate the performance of the proposed estimation algorithm and also to compare its performance with other known channel estimation techniques. Since the theoretical MSE expressions are not evaluated in paper, Monte Carlo simulation technique was used by running the simulation code for 1000 times, calculating the MSE every iteration and the result is averaged over all iterations to ensure that the obtained simulation results approaches the un-evaluated theoretical expressions. The time correlated channel was generated by an implementation of the Rosa. The generation of a time correlated channel impulse response is for every sample of the received and transmitted signal. As can be seen from the figures shown below that in all cases, the MSE of a channel estimation technique decrease with increasing SNR and increasing the FFT size as deduced in section 3 of the proposed channel estimation algorithm. Figure (2) Illustrates the MSE performance of the proposed channel estimator at different FFT size values. Figure (3) illustrates the MSE of the proposed channel estimation algorithm Vs. SNR at different values of the FFT size. It can be noticed that the FFT size has a dramatic effect on the value of the MSE at a given SNR. Current OFDM systems use an FFT size that is larger than 1024 which guarantees a MSE below 1e-4 even at low SNR values. Mean squared error performance of the proposed channel estimator vs. FFT size is depicted in Figure (3) at three different SNR values. This figure also emphasizes the same deduced inverse proportionality relation between the MSE and the FFT size. Figure (4) illustrates the MSE performance comparison between the proposed channel estimator and LS, LMMSE estimators for a user velocity of $60 \mathrm{~km} \mathrm{~h}^{-1}$ at FFT size $\mathrm{N}_{\mathrm{fft}}=64$. Figure (5) illustrates the MSE of the presented channel estimators for a user velocity of $60 \mathrm{~km} \mathrm{~h}^{-1}$ at FFT size $\mathrm{N}_{\mathrm{fft}}=128$ at a given SNR. Figure (6) illustrates the difference in MSE between the proposed estimator and the LMMSE estimator is decreasing as the FFT size increases from 64 to 256 .

Table 1. Simulation parameters

\begin{tabular}{ll}
\hline System & LTE \\
\hline Modulation & $16-\mathrm{QAM}$ \\
Symbol time & $224 \mu \mathrm{s}$ \\
Bandwidth & $5.71 \mathrm{MHz}$ \\
Speed & $60 \mathrm{~km} / \mathrm{hr}$ \\
FFT size & $64,128,256$ \\
Carrier frequency & $2.4 \mathrm{GHz}$ \\
Channel model & Rosa Zheng, dispersive, multipath \\
Channel estimators & LMMSE, LS Vs Proposed \\
\hline
\end{tabular}




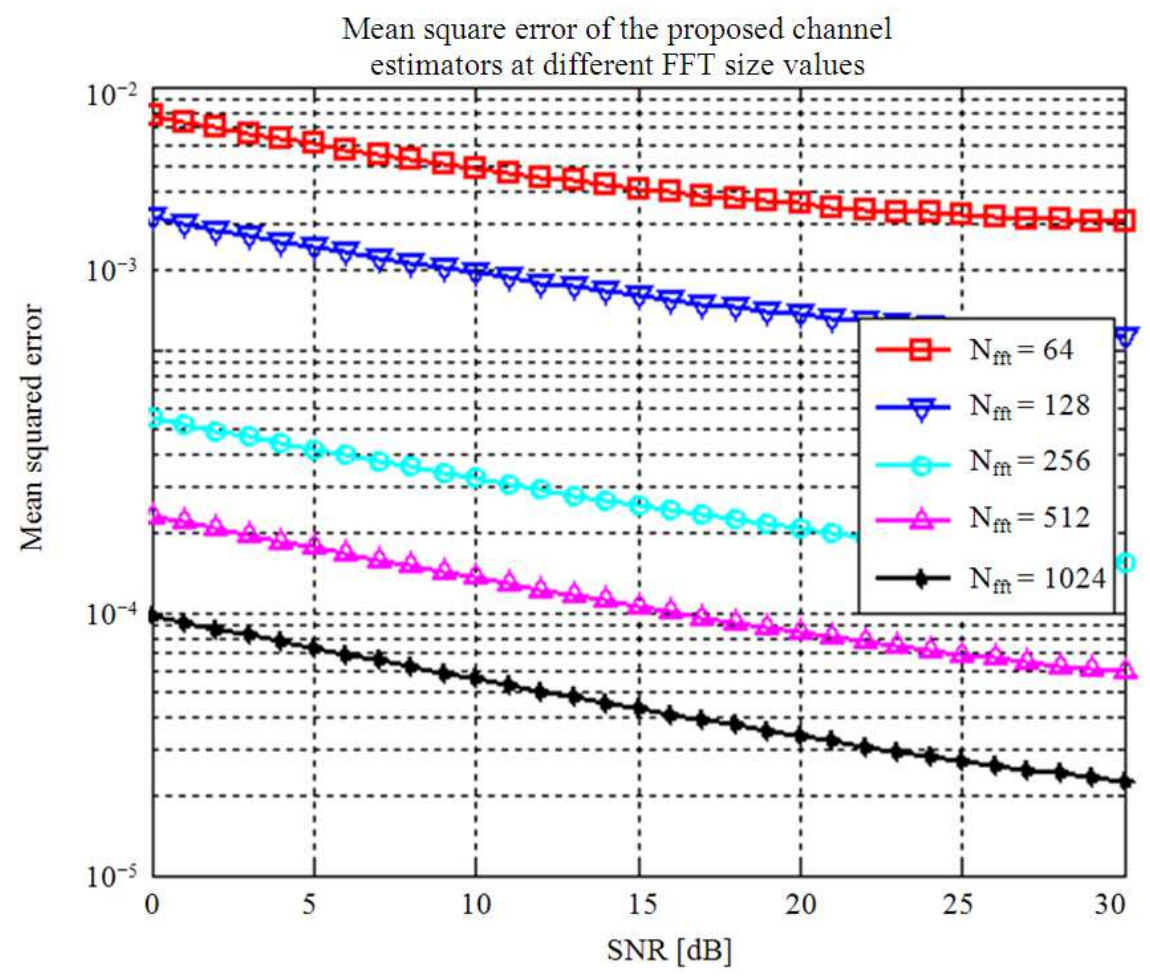

Figure (2) Mean squared error performance of theproposed channel estimator at different FFT size values

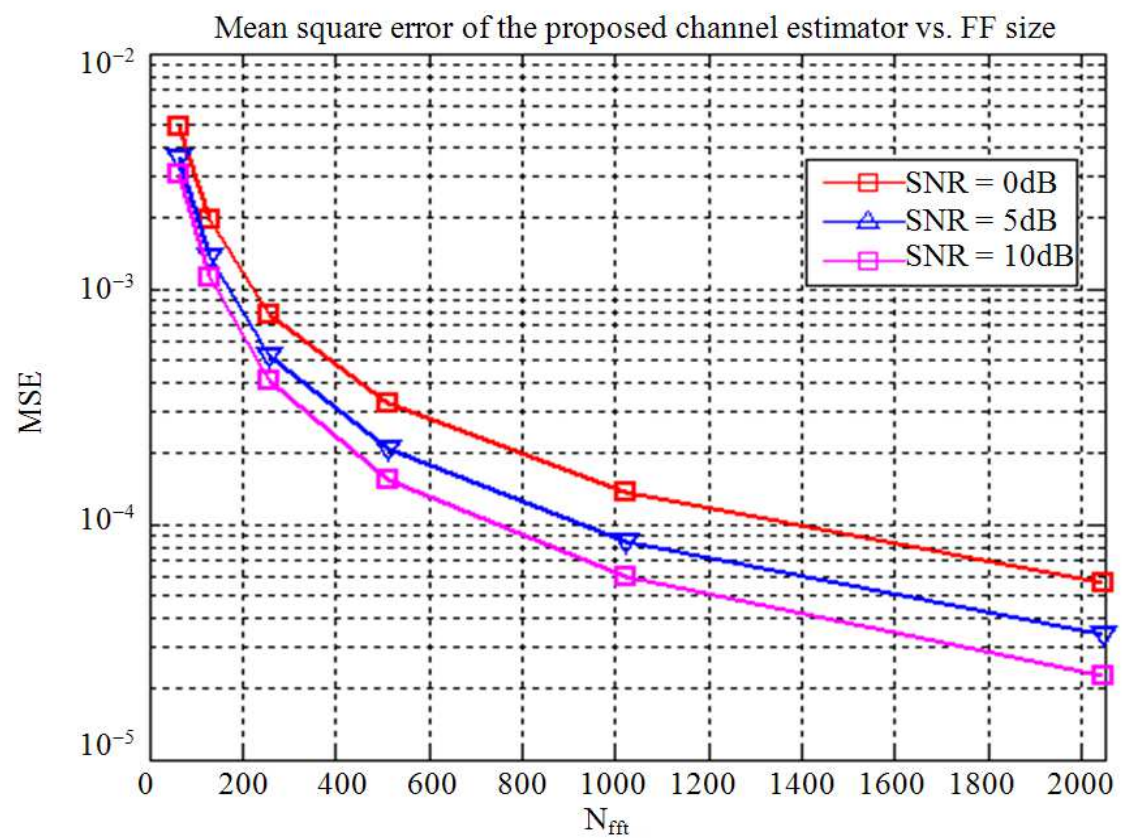

Figure (3) Mean squared error performance of the proposedchannel estimator Vs FFT size 


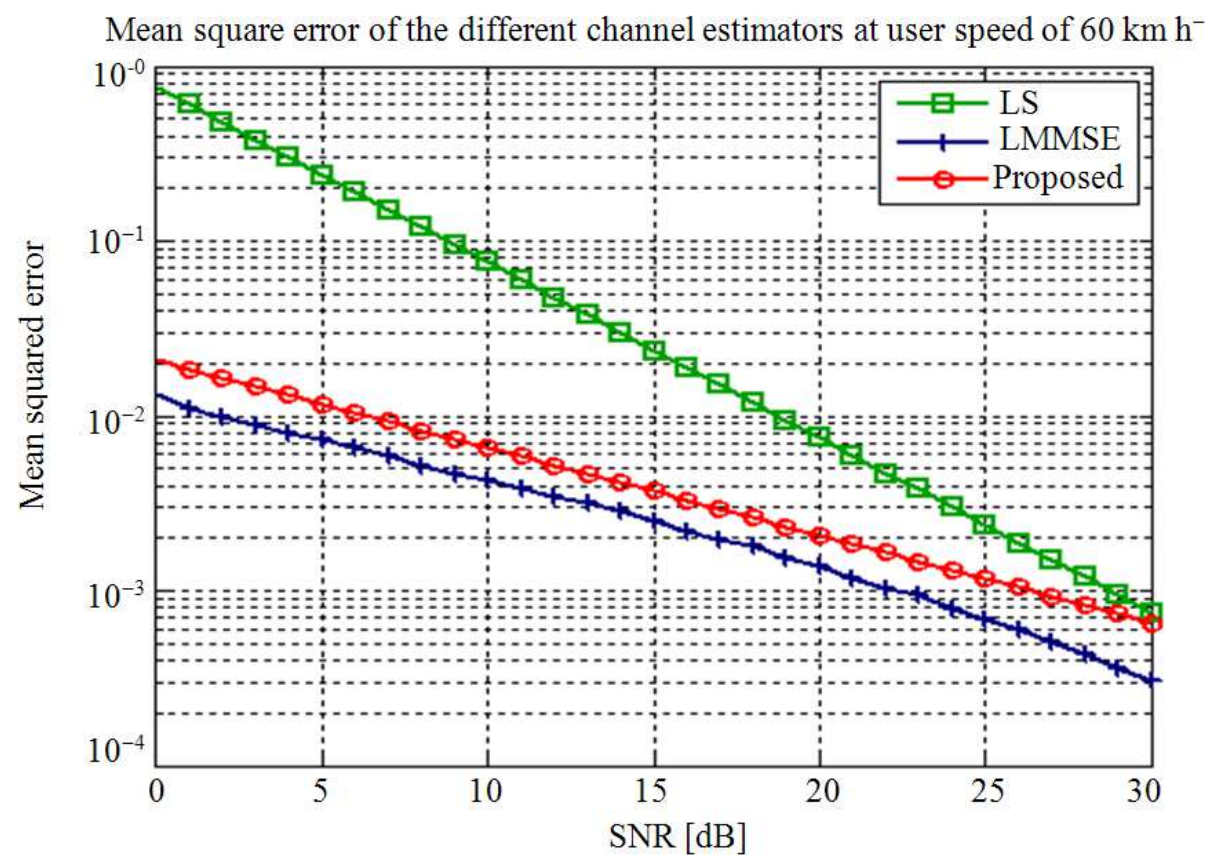

Figure (4) MSE performance comparison between the proposed channel estimator, LS and LMMSE estimators $\mathrm{N}_{\mathrm{fft}}=64$

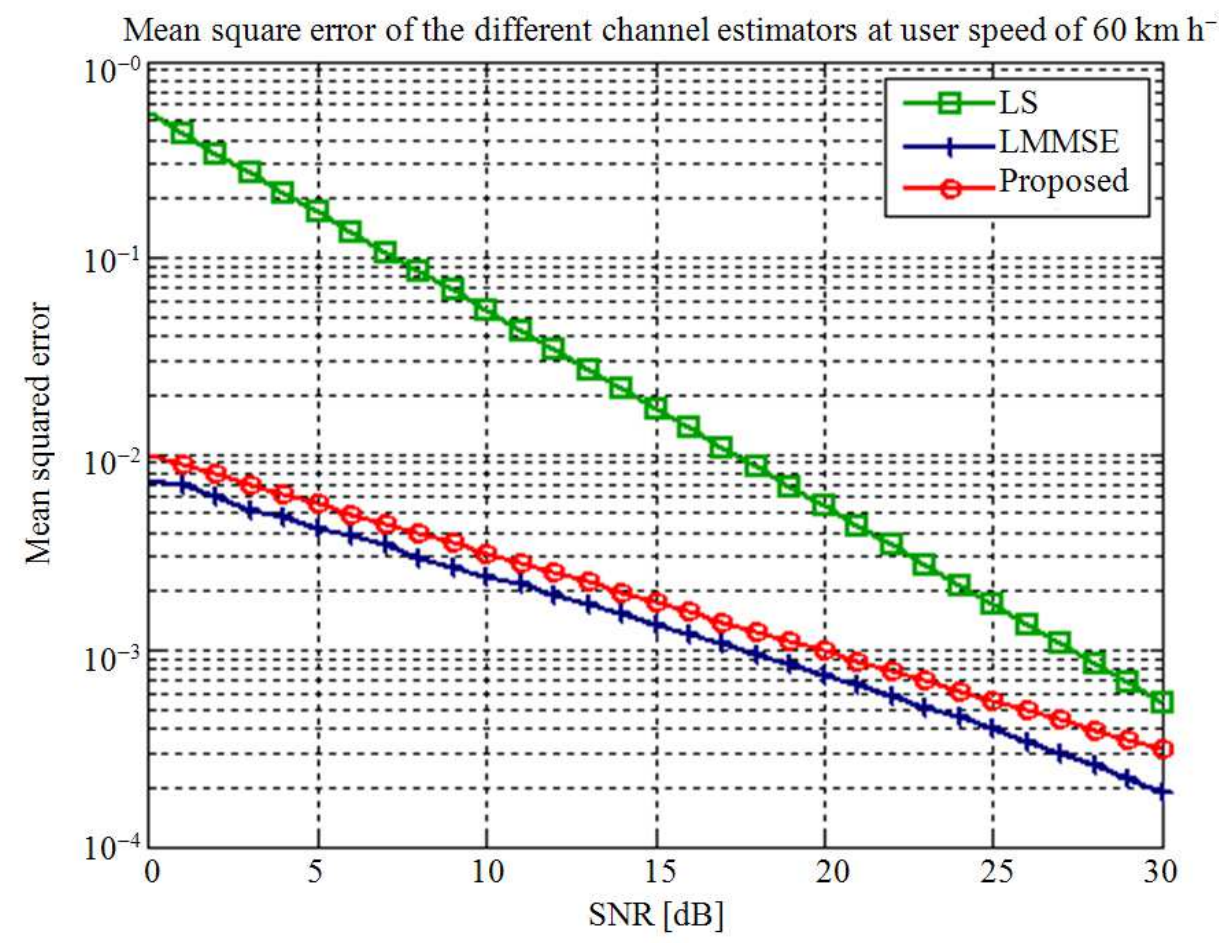

Figure (5) MSE performance comparison between the proposed channel estimator, LS and LMMSE estimators $\mathrm{N}_{\mathrm{fft}}=128$ 


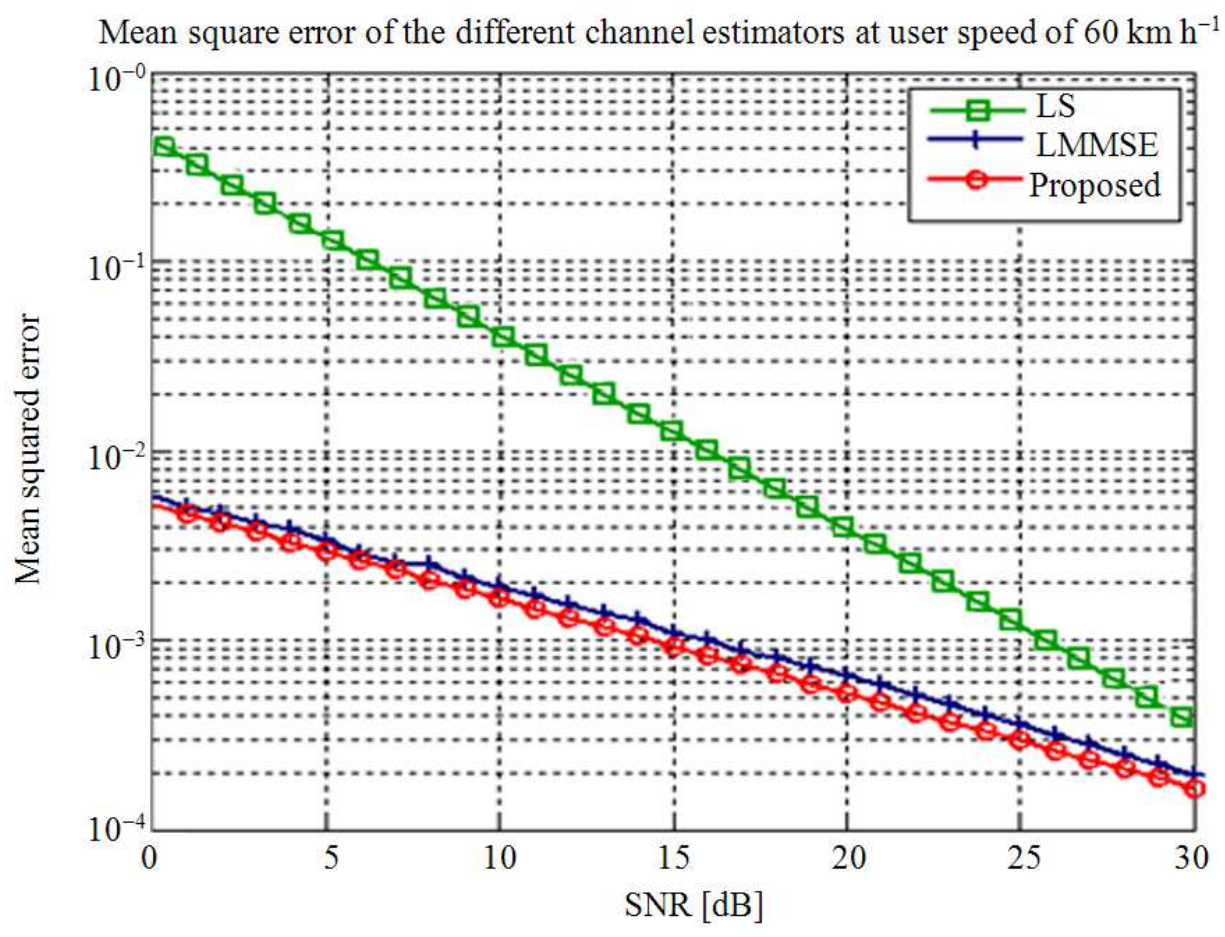

Figure (6) MSE performance comparison between the proposed channel estimator, LS and LMMSE estimators $\mathrm{N}_{\mathrm{fft}}=256$

\section{CONCLUSION}

A novel channel estimation algorithm has been proposed, evaluated and presented. This algorithm is based on Roza Zheng channel model and the matrix transpose rather than matrix inverse and it can be adopted to LTE systems. The proposed technique has the advantage of invariant complexity with respect to the channel matrix representing a dense multipath environment. The algorithm complexity is reduced due to the matrix multiplication process. Simulation results showed that the proposed algorithm is competitive to the optimum LMMSE from complexity point of view. The complexity reduction is compaired to LS and LMMSE algorithms. This baised on the accuracy considered when the MSE vesus the SNR are used as performance metrics of interest.

\section{REFERENCES}

Aziz, A.M., 2011. A soft-decision fusion approach for multiple-sensor distributed binary detection systems. IEEE Trans. Aeros. Electr. Syst., 47: 2208-2216. DOI: 10.1109/TAES.2011.5937293
Aziz, A.M., M. Ahmed, A. Mohamed and G.M.A. Hamid, 2011. A new soft fusion approach for multiple receivers wireless communication systems. Electr. Telecomm. Res. Instit. J., 33: 310-319.

Chen, Y., L. Cui and C. Qi, 2011. Performance analysis of pilot aided channel estimation methods for MBSFN in LTE system. Proceedings of the International Conference on Computational ProblemSolving, Oct. 21-23, IEEE Xplore Press, Chengdu, pp: 682-687. DOI: 10.1109/ICCPS.2011.6089767

Deng, B. and W. Wang, 2011. Low complexity channel estimation method of MIMO-OFDM for LTE downlink. Proceedings of the 7th International Conference on Wireless Communications, Networking and Mobile Computing, Sept. 23-25, IEEE Xplore Press, Wuhan, pp: 1-4. DOI: 10.1109/wicom.2011.6036656

Hong-Jin, W. and L. Li-Fa, 2011. A modified channel estimation based on svd in LTE-advanced systems. Proceedings of the 7th International Conference on Wireless Communications, Networking and Mobile Computing, Sept. 23-25, IEEE Xplore Press, Wuhan, pp: 1-5. DOI: 10.1109/wicom.2011.6040119 
Hou, J. and J. Liu, 2010. A novel channel estimation algorithm for 3GPP LTE downlink system using joint time-frequency two-dimensional iterative Wiener filter. Proceedings of the 12th IEEE International Conference on Communication Technology, Nov. 11-14, IEEE Xplore Press, Nanjing, pp: 289-292. DOI: 10.1109/ICCT.2010.5689239

Jiang, M., N. Prasad and X. Wang, 2010. Design of high performance MIMO receivers for LTE/LTE-a uplink. Proceedings of the 44th Asilomar Conference on Signals, Systems and Computers, Nov. 7-10, IEEE Xplore Press, Pacific Grove, CA., pp:

1493-1497. DOI: 10.1109/ACSSC.2010.5757785

Jing, S.X.Y. and Y. Fang, 2011. Channel estimation for TD-LTE system. Proccedings of the Cross Strait Quad-Regional Radio Science Wireless Technology Conference, Jul. 26-30, IEEE Xplore Press, Harbin, pp: 923-925.

DOI: 10.1109/CSQRWC.2011.6037107

Ketonen, J., M. Juntt and J.R. Cavallaro, 2009. Receiver implementation for MIMO-OFDM with AMC and precoding. Proceedings of the Conference Record of the 43th Asilomar Conference on Signals, Systems and Computers, Nov. 1-4, IEEE Xplore Press, Pacific Grove, CA., pp: 1268-1272. DOI: 10.1109/ACSSC.2009.5469950
Rana, M.M., 2010. Channel estimation techniques and LTE terminal implementation challenges. Proceedings of the 13th International Conference on Computer and Information Technology, Dec. 23-25, IEEE Xplore Press, Dhaka, pp: 545-549. DOI: 10.1109/ICCITECHN.2010.5723916

Schwarz, S., C. Mehlf and M. Rupp, 2010. Calculation of the spatial preprocessing and link adaption feedback for 3GPP UMTS/LTE. Proceedings of the 6th Conference on Wireless Advanced, Jun. 27-29, IEEE Xplore Press, London, pp: 1-6. DOI: 10.1109/WIAD.2010.5544947

Song, H., LY. Xiaowen and Yu. Ziming, 2011. A complexity reduction approach for LTE downlink channel estimation. Peoceeding of the International Conference on Internet Technology and Applications, Aug. 16-18, IEEE Xplore Press, Wuhan, pp: 1-4. DOI: 10.1109/ITAP.2011.6006127

Zhao, Q., Z. Zhang and Z. Shi, 2010. Researchlink simulation of key technologies for LTE downlink. Proceedings of the 6th International Conference (WiCOM) on (IEEE) Wireless Communications Networking Mobile Computing, Sept. 23-25, IEEE Xplore Press, Chengdu, pp: 1-4. DOI: 10.1109/WICOM.2010.5600207 